\title{
A performance comparison between two design techniques for non-linear output feedback control
}

\author{
C. XIE† and M. FRENCH†* \\ For a system possessing a non-linear output feedback normal form, an observer backstepping design is compared to a \\ high gain observer design with respect to non-singular performance cost functional. If the initial error between the initial \\ condition of the state and the initial condition of the observer is large, the high gain observer design is shown to have \\ better performance than the observer backstepping design. An output feedback system with parametric uncertainty is \\ then considered. It is shown that if an a priori estimate for the bound of the uncertain parameter is conservative, then an \\ adaptive observer backstepping design has better performance than the adaptive high gain observer design.
}

\section{Introduction}

In recent years several alternative constructive control techniques have been proposed for controlling non-linear systems using output feedback. In this paper we will be concerned with two major classes of control designs. The first class of controllers are based on high gain observers with saturated controls (see, e.g. Esfandiari and Khalil 1992, Khalil and Esfandiari 1993, Khalil 1996, Atassi and Khalil 1999). We refer to this class of control designs as Khalil designs. The second class of controllers are based on backstepping techniques (Kristic et al. 1995), and we refer to this class of controllers as $K K K$ designs.

The Khalil designs are applicable to affine systems of full relative degree, whilst the $K K K$ designs are applicable to an alternative class of systems, namely those which possess an output feedback normal form. By considering systems which are both full relative degree and have an output feedback normal form, we can compare the behaviour of the controllers on common systems. (Note also that such systems are characterized in a coordinate free manner (Kristic et al. 1995).) This motivates the study of comparison of different designs, as initiated in Khalil (1999).

The results in Khalil (1999) are purely numerical, and give rise to many interesting questions, such as:

- When are the Khalil designs more sensitive to disturbances than the $K K K$ designs, and vice versa?

- When do the $K K K$ designs require greater control effort than the Khalil designs, and vice versa?

- When do the Khalil designs have superior output transients to the $K K K$ designs, and vice versa?

Received 15 April 2003. Revised 4 December 2003.

* Author for correspondence. e-mail:mcf@ecs.soton.ac.uk

$\uparrow$ School of Electronics and Computer Science, University of Southampton, Southampton SO17 1BJ, UK.
In particular, by introducing suitable measures of performance and sensitivity we would like to be able to characterize situations in which one design is preferable to another. Such characterizations have obvious consequences for design choices, and also should give insight into the dynamics and trade-offs inherent in these controllers.

In this paper we will consider the latter two points, by considering a non-singular cost functional penalizing both the output transient and the control effort. It should be observed that whilst there are many results concerning the transient performance of the output (see, e.g. Kristic et al. 1995), there is little work in the literature on non-singular costs for non-optimal designs. See, however, French et al. (2000), French (2002) and Beleznay and French (2003) for related results and techniques.

In particular, for an output feedback system $\Sigma$ with input $u$ and output $y$, and a controller $\Xi$ mapping $y \mapsto u$, we consider the following cost which penalizes both the control and the output signal

$$
\begin{aligned}
P(\Sigma, \Xi) & =\int_{L^{2}\left(T_{\eta}\right)} y^{2} \mathrm{~d} t+\sup _{t \in \mathbb{R}_{+}}|u(t)| \\
& =\|y\|_{L^{2}\left(T_{\eta}\right)}^{2}+\|u\|_{L^{\infty}\left(\mathbb{R}_{+}\right)}
\end{aligned}
$$

where the time set $T_{\eta}$ is defined by

$$
T_{\eta}=\{t \geq 0|| y(t) \mid>\eta\}
$$

and $\eta$ is a small positive number. Such a cost penalizes the input and output response of the system whilst $y(t) \notin[-\eta, \eta]$, hence for a closed loop whose goal is to regulate $y$ to zero, keeping $y, u$ bounded, this cost is finite and is a reasonable penalty on the transient behaviour. Note that whilst a direct $L^{2}$ penalty on the output could be considered for the designs given, the relaxation of the output penalty is physically meaningful, and considerably simplifies the technical treatment.

In $\S 2$ we show that a Khalil design out-performs with a $K K K$ design when the information on initial state is poor and leads to a large initial observer error. In $\S 3$ we 
establish a result in the reverse direction. We consider an output feedback system with an unknown parameter, and then show that an adaptive $K K K$ design outperforms an adaptive Khalil design as the information on the size of the parameter becomes conservative.

\section{Performance of output feedback system}

In this section we study the performances of a $K K K$ design and a Khalil design for a system $\Sigma\left(x_{0}\right)$ which can be expressed in the output feedback form

$$
\begin{aligned}
\Sigma\left(x_{0}\right): \quad \dot{x} & =A x+\varphi(y)+B u, \quad x(0)=x_{0} \\
y & =C x
\end{aligned}
$$

where

$$
\begin{aligned}
& x=\left(\begin{array}{c}
x_{1} \\
x_{2} \\
\vdots \\
x_{n}
\end{array}\right), \quad x_{0}=\left(\begin{array}{c}
x_{01} \\
x_{02} \\
\vdots \\
x_{0 n}
\end{array}\right), \quad \varphi(y)=\left(\begin{array}{c}
\varphi_{1}(y) \\
\varphi_{2}(y) \\
\vdots \\
\varphi_{n}(y)
\end{array}\right) \\
& A=\left(\begin{array}{cccccc}
0 & 1 & 0 & \cdots & 0 & 0 \\
0 & 0 & 1 & \cdots & 0 & 0 \\
\cdots & 0 & 0 & \cdots & 0 & 0 \\
0 & 0 & 0 & \cdots & 0 & 0
\end{array}\right), \quad B=\left(\begin{array}{c}
0 \\
\vdots \\
0 \\
1
\end{array}\right), \\
& C=(1,0, \ldots, 0)
\end{aligned}
$$

and $u$ is the control input, $y$ is the measured output, $x_{0}$ is the initial condition of the state, and the functions $\varphi_{i}$ are sufficiently smooth and Lipschitz continuous, moreover, we assume that $\varphi_{i}(0)=0, i=1, \ldots, n$ through this paper.

\subsection{Initialization of the observer}

Let us first consider a generic observer based controller $\Xi\left(\hat{x}_{0}\right)$, where $\hat{x}_{0}$ is the initial condition for the observer. The performance of the closed loop $\left(\Sigma\left(x_{0}\right), \Xi\left(\hat{x}_{0}\right)\right)$ is dependent on both the initial state $x_{0}$ and the initial condition for the observer $\hat{x}_{0}$. Whilst the initial state $x_{0}$ is the property of a system, the control designer has the freedom to choose the initial condition $\hat{x}_{0}$ for the observer.

It is intuitive that good performance results from initializing the observer state $\hat{x}_{0}$ to be close to the actual initial state $x_{0}$. Of course, in practice, the initial state is often unknown, so it can be hard to initialize in this manner. Nevertheless standard practice is to try to minimize

$$
\left\|\tilde{x}_{0}\right\|=\left\|x_{0}-\hat{x}_{0}\right\|
$$

according to the best information available. To establish a rigorous justification for this intuitive idea (or more precisely: to characterize the situations when it is valid) remains an open research problem; in this paper we simply illustrate the validity of this approach on a single example, as discussed next.

Consider the two-dimensional system

$$
\begin{aligned}
\Sigma^{0}\left(x_{0}\right): \quad \dot{x}_{1} & =x_{2} \\
\dot{x}_{2} & =\varphi(y)+u, \quad x(0)=\left(x_{01}, x_{02}\right)^{\mathrm{T}} \\
y & =x_{1}
\end{aligned}
$$

where $\varphi(y)$ is a Lipschitz continuous function. We consider a $K K K$ controller (see Kristic et al. 1995; Ch. 7, p. 291) defined as

$$
\begin{aligned}
\Xi_{O}^{0}\left(\hat{x}_{0}\right): & u=\alpha_{2}\left(y, \hat{x}_{1}, \hat{x}_{2}\right) \\
\dot{\hat{x}}_{1} & =\hat{x}_{2}+k_{1}\left(y-\hat{x}_{1}\right) \\
\dot{\hat{x}}_{2} & =k_{2}\left(y-\hat{x}_{1}\right)+\varphi(y)+u, \quad \hat{x}(0)=\left(\hat{x}_{01}, \hat{x}_{02}\right)^{\mathrm{T}}
\end{aligned}
$$

where

$$
\begin{aligned}
\xi_{1}(y) & =y \\
\alpha_{1}(y)= & -c_{1} \xi_{1}-d_{1} \xi_{1} \\
\xi_{2}\left(y, \hat{x}_{1}, \hat{x}_{2}\right)= & \hat{x}_{2}-\alpha_{1}\left(y, \hat{x}_{1}\right) \\
\alpha_{2}\left(y, \hat{x}_{1}, \hat{x}_{2}\right)= & -c_{2} \xi_{2}-\xi_{1}-d_{2}\left(\frac{\partial \alpha_{1}}{\partial y}\right)^{2} \xi_{2} \\
& -k_{2}\left(y-\hat{x}_{1}\right)-\varphi(y)+\frac{\partial \alpha_{1}}{\partial y} \hat{x}_{2}
\end{aligned}
$$

and $k_{1}, k_{2}, c_{i}, d_{i}, 1 \leq j \leq 2$ are positive constants.

Since we can measure $x_{1}$, we can always take $\hat{x}_{01}=x_{01}$. However, $x_{02}$ may be unknown, and so it is meaningful to compare the behaviour of the closed loops with the alternative choices of

$$
\hat{x}_{02}=x_{02}, \quad \hat{x}_{02}=0
$$

We can then show the following proposition, whose proof is given in the Appendix.

Proposition 1: Consider the system $\Sigma^{0}\left(x_{0}\right)$ and the controller $\Xi^{0}\left(\hat{x}_{0}\right)$, then there exist $c_{i}, d_{i}, k_{i}(i=1,2)$ such that

$$
\begin{aligned}
\lim _{x_{02} \rightarrow \infty} & \left(P\left(\Sigma^{0}\left(x_{0}\right), \Xi_{O}^{0}\left(\left(x_{01}, 0\right)^{\mathrm{T}}\right)\right)\right. \\
& \left.-P\left(\Sigma^{0}\left(x_{0}\right), \Xi_{O}^{0}\left(\left(x_{01}, x_{02}\right)^{\mathrm{T}}\right)\right)\right)=+\infty
\end{aligned}
$$

This proposition shows that as $x_{02}$ becomes large, the difference of performance between $P\left(\Sigma^{0}\left(x_{0}\right), \Xi_{O}^{0} \times\right.$ $\left.\left(\left(x_{01}, 0\right)^{\mathrm{T}}\right)\right)$ and $P\left(\Sigma^{0}\left(x_{0}\right), \Xi_{O}^{0}\left(\left(x_{01}, x_{02}\right)^{\mathrm{T}}\right)\right)$ can be larger than any positive constant. Therefore, it is advantageous to initialize the second state of the observer close to actual state rather than to initialize it at zero.

However, we may well not possess complete information concerning the value of the initial condition of the state, that is we do not exactly know $x_{0}$, and hence we have to take $\hat{x}_{0}$ to be the best estimate to $x_{0}$. Then we 
are interested in studying the situation in which our estimate of $x_{0}$ is not accurate and $\left\|\tilde{x}_{0}\right\|$ is large, in particular how does poor information on $x_{0}$ (which causes 'bad' choices of $\hat{x}_{0}$ ), affect the performance of the controllers?

\subsection{KKK design}

We first consider a $K K K$ design (Kristic et al. 1995) which achieves global regulation of the output. Although the $K K K$ design has a global region of attraction (in $\left(x_{0}, \hat{x}_{0}\right)$ ), we will prove that the performance of the controller can degrade arbitrarily as the initial error $\left\|\tilde{x}_{0}\right\|$ becomes large for any fixed initial state condition $x_{0}$.

The $K K K$ design (Kristic et al. 1995) for system $\Sigma\left(x_{0}\right)$ is as follows.

First, an observer is defined by

$$
\begin{aligned}
& \dot{\hat{x}}=A \hat{x}+k(y-\hat{y})+\varphi(y)+B u, \quad \hat{x}(0)=\hat{x}_{0} \\
& \hat{y}=C \hat{x}
\end{aligned}
$$

where

$$
k=\left(k_{1}, k_{2}, \ldots, k_{n}\right)^{\mathrm{T}}, \quad k_{i}>0, \quad 1 \leq i \leq n
$$

is chosen such that $A-k C$ is Hurwitz.

Then define

$$
\begin{aligned}
\xi_{1}(y)= & y \\
\alpha_{1}(y)= & -c_{1} \xi_{1}-d_{1} \xi_{1}-\varphi_{1}(y) \\
\xi_{i}\left(y, \hat{x}_{1}, \ldots, \hat{x}_{i}\right)= & \hat{x}_{i}-\alpha_{i-1}\left(y, \hat{x}_{1}, \ldots, \hat{x}_{i-1}\right) \\
\alpha_{i}\left(y, \hat{x}_{1}, \ldots, \hat{x}_{i}\right)= & -c_{i} \xi_{i}-\xi_{i-1}-d_{i}\left(\frac{\partial \alpha_{i-1}}{\partial y}\right)^{2} \xi_{i} \\
& -k_{i}\left(y-\hat{x}_{1}\right)-\varphi_{i}(y) \\
& +\frac{\partial \alpha_{i-1}}{\partial y}\left(\hat{x}_{2}+\varphi_{1}(y)\right) \\
& +\sum_{j=1}^{i-1} \frac{\partial \alpha_{i-1}}{\partial \hat{x}_{j}}\left(\hat{x}_{j+1}+k_{j}\left(y-\hat{x}_{1}\right)+\varphi_{j}(y)\right) \\
i= & 2,3, \ldots, n
\end{aligned}
$$

where $c_{i}, d_{i}, 1 \leq i \leq n$ are positive constants. The controller is then defined as

$$
\begin{aligned}
\Xi_{O}\left(\hat{x}_{0}\right): \quad & u=\alpha_{n}\left(y, \hat{x}_{1}, \ldots, \hat{x}_{n}\right) \\
& \dot{\hat{x}}=A \hat{x}+k(y-\hat{y})+\varphi(y)+B u, \quad \hat{x}(0)=\hat{x}_{0} \\
\hat{y} & =C \hat{x}
\end{aligned}
$$

The following result summarizes the standard properties of this closed loop.

Proposition 2: Consider the closed loop system $\left(\Sigma\left(x_{0}\right)\right.$, $\left.\Xi_{O}\left(\hat{x}_{0}\right)\right)$. For any initial data $x_{0} \in \mathbb{R}^{n}$ and $\hat{x}_{0} \in \mathbb{R}^{n}$, the following hold:

(1) The signals $x, \hat{x}, u$ and $y$ and bounded;
(2) The output is regulated to zero

$$
\lim _{t \rightarrow \infty} y(t)=0
$$

(3) The performance is finite

$$
P\left(\Sigma\left(x_{0}\right), \Xi_{O}\left(\hat{x}_{0}\right)\right)<\infty
$$

Proof: The proof of 1, 2 can be found in Kristic et al. (1995). Let $m\left(T_{\eta}\right)$ denote the Lebesgue measure of the set $T_{\eta}$. Note that $m\left(T_{\eta}\right)<\infty$ since $y(t) \rightarrow 0$ as $t \rightarrow \infty$ hence

$$
\|y\|_{L^{2}\left(T_{\eta}\right)} \leq m\left(T_{\eta}\right)^{1 / 2}\|y\|_{L^{\infty}\left(\mathbb{R}_{+}\right)}<\infty
$$

by 1 . The boundedness of the performance follows directly.

We now establish the critical performance property for the $K K K$ design, which states that the performance gets arbitrarily large as the initial observer error increases.

Theorem 1: For any choice of the controller gains $k_{i}$, $1 \leq i \leq n$, and for any fixed initial state $x_{0}$ of the system $\Sigma\left(x_{0}\right)$, the performance of the controller $\Xi_{O}\left(\hat{x}_{0}\right)$ has the property

$$
\limsup _{\left\|\tilde{x}_{0}\right\| \rightarrow \infty} P\left(\Sigma\left(x_{0}\right), \Xi_{O}\left(\hat{x}_{0}\right)\right)=\infty
$$

Proof: For the convenience of notation, the following definitions are introduced

$$
\begin{gathered}
\xi_{i}(0)=\left.\xi_{i}\left(y, \hat{x}_{1}, \ldots, \hat{x}_{i}\right)\right|_{t=0} \\
\alpha_{i}(0)=\left.\alpha_{i}\left(y, \hat{x}_{1}, \ldots, \hat{x}_{i}\right)\right|_{t=0} \\
j=1,2, \ldots, n
\end{gathered}
$$

To prove this theorem, it suffices to show

$$
\limsup _{\left\|\tilde{x}_{0}\right\| \rightarrow \infty}\|u\|_{L^{\infty}\left(\mathbb{R}_{+}\right)}=\infty
$$

Since $u(t)$ is continuous, to establish the above equation, we only need to show

$$
\limsup _{\left\|\tilde{x}_{0}\right\| \rightarrow \infty} u(0)=\limsup _{\left\|\tilde{x}_{0}\right\| \rightarrow \infty} \alpha_{n}(0)=\infty
$$

Let $C \subset \mathbb{R}^{n-1}$ be a compact set, define

$$
C_{r}=\left\{\hat{x}_{0} \in \mathbb{R}^{n} \mid\left(\hat{x}_{01}, \ldots, \hat{x}_{0, n-1}\right) \in C ; \hat{x}_{0 n}=r\right\}
$$

Consider the initial data of the observer $\hat{x}_{0} \in C_{r}$. Because $x_{0}$ is fixed, if we can prove that

$$
\lim _{r \rightarrow \infty} \sup _{\hat{x}_{0} \in C_{r}} \alpha_{n}(0)=\infty
$$

then (4) will hold. 
We now establish (5). Since all $\varphi_{i}$ and its derivatives are continuous functions it follows that $\alpha_{i}$ and $\xi_{i}$ are continuous functions of their variables. Note that

$$
\begin{aligned}
\xi_{i}(0)= & \hat{x}_{0 i}-\alpha_{i-1}(0) \\
\alpha_{i}(0)= & -c_{i} \xi_{i}(0)-\xi_{i-1}(0)-d_{i}\left(\left.\frac{\partial \alpha_{i-1}}{\partial y}\right|_{t=0}\right)^{2} \xi_{i}(0) \\
& -k_{i}\left(x_{01}-\hat{x}_{01}\right)-\varphi_{i}\left(x_{01}\right) \\
& +\sum_{j=1}^{i-1}\left(\left.\frac{\partial \alpha_{i-1}}{\partial \hat{x}_{j}}\right|_{t=0}\right)\left(\hat{x}_{0, j+1}+k_{j}\left(x_{01}-\hat{x}_{01}\right)+\varphi_{j}\left(x_{01}\right)\right)
\end{aligned}
$$

So, for $1 \leq i \leq n-1, \xi_{i}(0), \alpha_{i}(0)$ are independent of $\hat{x}_{0 n}$, i.e. bounded independently of $r$. Therefore there exists $M>0$ dependent on $C$ and $x_{01}$ but not on $r$, for which $\sup _{\hat{x}_{0} \in C_{r}}\left|\xi_{i}(0)\right| \leq M, \quad \sup _{\hat{x}_{0} \in C_{r}}\left|\alpha_{i}(0)\right| \leq M, \quad 1 \leq i \leq n-1$ Now we compute $\alpha_{n}(0)$. First, we have

$$
\xi_{n}(0)=\hat{x}_{0 n}-\alpha_{n-1}(0)=r-\alpha_{n-1}(0)
$$

and so

$$
\begin{aligned}
\alpha_{n}(0)= & -c_{i} \xi_{n}(0)-\xi_{n-1}(0)-d_{n}\left(\left.\frac{\partial \alpha_{n-1}}{\partial y}\right|_{t=0}\right)^{2} \xi_{n}(0) \\
& -k_{n}\left(x_{01}-\hat{x}_{01}\right)-\varphi_{n}\left(x_{01}\right)+\sum_{j=1}^{n-1}\left(\left.\frac{\partial \alpha_{n}-1}{\partial \hat{x}_{j}}\right|_{t=0}\right) \\
& \times\left(\hat{x}_{0, j+1}+k_{j}\left(x_{01}-\hat{x}_{01}\right)+\varphi_{j}\left(x_{01}\right)\right) \\
= & \left(-c_{n}-d_{n}\left(\left.\frac{\partial \alpha_{n-1}}{\partial y}\right|_{t=0}\right)^{2}\right) r+\left(\left.\frac{\partial \alpha_{n-1}}{\partial_{n-1}}\right|_{t=0}\right) r \\
& +F\left(x_{01}, \hat{x}_{01}, \ldots, \hat{x}_{0, n-1}\right)
\end{aligned}
$$

where

$$
\begin{aligned}
& F\left(x_{01}, \hat{x}_{01}, \ldots, \hat{x}_{0, n-1}\right) \\
& =\left(c_{n}+d_{n}\left(\left.\frac{\partial \alpha_{n-1}}{\partial y}\right|_{t=0}\right)^{2}-\left.\frac{\partial \alpha_{n-1}}{\partial \hat{x}_{n-1}}\right|_{t=0}\right) \alpha_{n-1}(0) \\
& \quad+\xi_{n-1}(0)-k_{n}\left(x_{01}-\hat{x}_{01}\right)-\varphi_{n}\left(x_{01}\right) \\
& \quad+\sum_{j=1}^{n-2}\left(\left.\frac{\partial \alpha_{n-1}}{\partial \hat{x}_{j}}\right|_{t=0}\right)\left(\hat{x}_{0, j+1}+k_{j}\left(x_{01}-\hat{x}_{01}\right)+\varphi_{j}\left(x_{01}\right)\right)
\end{aligned}
$$

is independent of $\hat{x}_{0 n}$, namely $r$. Let us consider the second term of the expression for $\alpha_{n}(0)$.

$$
\begin{aligned}
\frac{\partial \alpha_{i}}{\partial \hat{x}_{i}} & =-c_{i} \frac{\partial \xi_{i}}{\partial \hat{x}_{i}}-d_{i}\left(\frac{\partial \alpha_{i-1}}{\partial y}\right)^{2} \frac{\partial \xi_{i}}{\partial \hat{x}_{i}}+\frac{\partial \alpha_{i-1}}{\partial \hat{x}_{i-1}} \\
& =-c_{i}-d_{i}\left(\frac{\partial \alpha_{i-1}}{\partial y}\right)^{2}+\frac{\partial \alpha_{i-1}}{\partial \hat{x}_{i-1}} .
\end{aligned}
$$

Therefore, by recursive substitution we obtain

$$
\begin{aligned}
\frac{\partial \alpha_{n-1}}{\partial \hat{x}_{n-1}} & =\sum_{j=2}^{n-1}\left(-c_{j}-d_{j}\left(\frac{\partial \alpha_{j-1}}{\partial y}\right)^{2}\right)+\frac{\partial \alpha_{1}}{\partial \hat{x}_{1}} \\
& =\sum_{j=2}^{n-1}\left(-c_{j}-d_{j}\left(\frac{\partial \alpha_{j-1}}{\partial y}\right)^{2}\right)
\end{aligned}
$$

since $\alpha_{1}$ is independent of $\hat{x}_{1}$.

Hence,

$$
\begin{aligned}
\alpha_{n}(0)= & r \sum_{j=2}^{n}\left(-c_{j}-d_{j}\left(\left.\frac{\partial \alpha_{j-1}}{\partial y}\right|_{t=0}\right)^{2}\right) \\
& +F\left(x_{01}, \hat{x}_{01}, \ldots, \hat{x}_{0, n-1}\right) .
\end{aligned}
$$

Because $c_{j}$ and $d_{j}$ are all positive numbers, and $F$ is independent of $r$, this establishes (5) as required.

\subsection{Khalil design}

It is well known that by a suitable coordinate transformation the system $\Sigma\left(x_{0}\right)$ can also be written as integrator chain with a matched nonlinearity. Concretely, we define a coordinate transformation

$$
T: \mathbb{R}^{n} \rightarrow \mathbb{R}^{n}, \quad z=T(x)
$$

by

$$
\begin{aligned}
T: & z_{1}=x_{1}, z_{2}=x_{2}+\psi_{1}\left(x_{1}\right), \ldots, \\
z_{n} & =x_{n}+\psi_{n-1}\left(x_{1}, x_{2}, \ldots, x_{n-1}\right)
\end{aligned}
$$

where

$$
\begin{gathered}
\psi_{i}\left(x_{1}, \ldots, x_{i}\right)=\varphi_{i}\left(x_{1}\right)+\sum_{j=1}^{i-1} \frac{\partial \psi_{i-1}}{\partial x_{j}}\left(x_{j+1}+\varphi_{j}\left(x_{1}\right)\right), \\
1 \leq i \leq n
\end{gathered}
$$

Then in the $z$ coordinates, $\Sigma\left(x_{0}\right)$ is of the form

$$
\begin{aligned}
\Sigma\left(z_{0}\right): \quad \dot{z} & =A z+B(\psi(z)+u), \quad z(0)=z_{0} \\
y & =C z
\end{aligned}
$$

where

$$
\left.\begin{array}{rl}
z_{0} & =T\left(x_{0}\right) \\
\psi(z) & =\psi_{n}\left(T^{-1}(z)\right) \\
\psi_{n}(x) & =\psi_{n}\left(x_{1}, \ldots, x_{n}\right)
\end{array}\right\}
$$

Since the system $\Sigma\left(x_{0}\right)$ has a uniform relative degree $n$, the mapping $T$ is a global diffeomorphism in $\mathbb{R}^{n}$; see Isidori (1989). Hence the inverse mapping $T^{-1}$ exists.

Remark 1: Since the output $y$ is unchanged by the transformation $T$, and the control input $u$ is independent of the change of variables, the performance $P$ is independent of $T$. 
Hence the Khalil designs considered in Esfandiari and Khalil (1992), Khalil and Esfandiari (1993) and Atassi and Khalil (1999) can be applied to the system $\Sigma\left(z_{0}\right)$. Typical results establish semiglobal regulation of the output. The Khalil designs utilize a high gain observer and a nonlinear separation principle (Atassi and Khalil 1999) which allow the observer and a globally bounded state feedback controller to be designed separately, and then combined using certainty equivalence, to ensure semiglobal results and closeness of the output feedback controller's trajectory to the underlying state feedback controller's trajectory. For the system $\Sigma\left(x_{0}\right)$, if $\varphi_{i}$ and its higher derivatives are globally bounded, it is straightforward to design a globally bounded state feedback controller achieving bounded performance. Hence through the high gain observer we can design an output feedback controller, which, for fixed initial condition of the state $z_{0}=T\left(x_{0}\right)$ and any initial condition of the observer $\hat{z}_{0}$ also has bounded performance. Furthermore, if the initial error

$$
\left\|\tilde{z}_{0}\right\|=\left\|z_{0}-\hat{z}_{0}\right\|
$$

becomes large, this design still achieves a bounded performance independent of the initial condition of the observer.

To design an output feedback controller, we first give a state feedback controller for $\Sigma\left(x_{0}\right)$. The controller

$$
u=-\psi(z)+v
$$

feedback linearizes the system $\Sigma\left(x_{0}\right)$, yielding

$$
\begin{aligned}
& \dot{z}=A z+B v, \quad z(0)=z_{0} \\
& y=C z
\end{aligned}
$$

We first design a bounded state feedback controller for the linear system (9). From Sussmann et al. (1994) we have the following lemma.

Lemma 1: The system (9) is null controllable with bounded control $(A N C B C)$ if and only if:

(1) A has no eigenvalues with positive real part;

(2) The pair $(A, B)$ is stabilizable in the ordinary sense.

Now since all the eigenvalues of $A$ are zero, namely, without positive real parts, and the pair $(A, B)$ is stabilizable, the system (9) is null controllable with bounded control, and, furthermore, there exists bounded state feedback controllers for the system (9). An explicit example (Sussmann et al. 1994) of such a bounded state feedback controller is given by

$$
v=-\sum_{i=1}^{n} \delta^{i} \operatorname{sat}\left(h_{i}(z)\right)
$$

where $0<\delta \leq \frac{1}{4}$, each $h_{i}: \mathbb{R}^{n} \rightarrow \mathbb{R}, 1 \leq i \leq n$, is a linear function, and sat(.) is the saturation function defined by

$$
\operatorname{sat}(w)=\left\{\begin{array}{cl}
-1, & w<-1 \\
w, & -1 \leq w \leq 1 \\
1, & w>1
\end{array}\right.
$$

This controller achieves global asymptotic stability for the resulting closed-loop system (Sussmann et al. 1994).

Consequently, the state feedback controller

$$
\Xi_{s}: \quad u=-\psi(z)-\sum_{i=1}^{n} \delta^{i} \operatorname{sat}\left(h_{i}(z)\right)
$$

globally asymptotically stabilizes the origin of system $\Sigma\left(z_{0}\right)$.

Now we design a output feedback controller for $\Sigma\left(x_{0}\right)$. Following Esfandiari and Khalil (1992) and Atassi and Khalil (1999), we define the high gain observer as

$$
\dot{\hat{z}}=A \hat{z}+H\left(y-\hat{z}_{1}\right), \quad \hat{z}(0)=\hat{z}_{0}
$$

where

$$
H=H(\epsilon)=\left(\frac{\alpha_{1}}{\epsilon}, \frac{\alpha_{2}}{\epsilon^{2}}, \ldots, \frac{\alpha_{n}}{\epsilon^{n}}\right)^{\mathrm{T}}
$$

and $\epsilon$ is a positive constant to be specified. The positive constants $\alpha_{i}, 1 \leq i \leq n$, are chosen such that the roots of the equation

$$
s^{n}+\alpha_{1} s^{n-1}+\cdots+\alpha_{n-1} s+\alpha_{n}=0
$$

are in the open left-half plane.

To apply the non-linear separation principle, the state feedback controller is required to be globally bounded. Generally, this property can be achieved by saturating the controller outside some set. But in our case we are interested in the initial condition of the observer becoming large. Instead, we introduce further assumptions on $\varphi_{i}$ to ensure that $\psi$ is globally bounded.

Lemma 2: For system $\Sigma\left(x_{0}\right)$, suppose $\varphi_{i} \in C^{n-i}(\mathbb{R})$, $\varphi_{i}^{(k)} \in L^{\infty}(\mathbb{R}), 1 \leq i \leq n ; 1 \leq k \leq n$, then $\psi$ defined by (7) lies in $L^{\infty}\left(\mathbb{R}^{n}\right)$.

Proof: Since $\varphi_{i} \in C^{n-i}(\mathbb{R}), \varphi_{i}^{(k)} \in L^{\infty}(\mathbb{R})$, from (6) we have that $\psi_{n}(x)$ is continuous and in $L^{\infty}\left(\mathbb{R}^{n}\right)$. Note that the mapping $T$ is a global diffeomorphism, we know that $\psi(z)$ also is continuous and in $L^{\infty}\left(\mathbb{R}^{n}\right)$.

Suppose that the conditions of Lemma 2 are satisfied, then the state feedback controller (11) is globally bounded, so an output feedback controller for system $\Sigma\left(x_{0}\right)$ can be taken as

$$
\begin{aligned}
\Xi_{H(\epsilon)}\left(\hat{z}_{0}\right): \quad & u=-\psi(\hat{z})-\sum_{i=1}^{n} \delta^{i} \operatorname{sat}\left(h_{i}(\hat{z})\right) \\
\dot{\hat{z}} & =A \hat{z}+H\left(y-\hat{z}_{1}\right), \quad \hat{z}(0)=\hat{z}_{0}
\end{aligned}
$$


For the system $\Sigma\left(z_{0}\right)$ and the output feedback controller $\Xi_{H(\epsilon)}\left(\hat{z}_{0}\right)$, relevant properties of the closed loop are summarized below.

Proposition 3: For system $\Sigma\left(z_{0}\right)$, suppose that $z_{0}=$ $T\left(x_{0}\right), x_{0}$ is fixed, and the assumption of Lemma 2 is satisfied. Then for any $\tilde{z}_{0}=z_{0}-\hat{z}_{0}$ there exists $\epsilon^{*}$ such that for all $\epsilon: 0<\epsilon<\epsilon^{*}$ the output feedback controller $\Xi_{H(\epsilon)}\left(\hat{z}_{0}\right)$ guarantees:

(1) The signals $z, \hat{z}, u$ and $y$ are bounded;

(2) The output is regulated to zero

$$
\lim _{t \rightarrow \infty} y(t)=0
$$

(3) The following limit

$$
\lim _{\epsilon \rightarrow 0} z(t, \epsilon)=\bar{z}(t)
$$

holds uniformly in $t$ for all $t \geq 0$, where $z(t, \epsilon)$ is the solution of the closed system $\left(\Sigma\left(z_{0}\right), \Xi_{H(\epsilon)}\left(\hat{z}_{0}\right)\right)$; and $\bar{z}(t)$ is the solution of the state feedback control closed system $\left(\Sigma\left(z_{0}\right), \Xi_{s}\right)$.

(4) The performance is finite

$$
P\left(\Sigma\left(z_{0}\right), \Xi_{H(\epsilon)}\left(\hat{z}_{0}\right)\right)<\infty
$$

Proof: First, the function

$$
\pi(z)=-\psi(z)-\sum_{i=1}^{n} \delta^{i} \operatorname{sat}\left(h_{i}(z)\right)
$$

is locally Lipschitz continuous since $\psi(z)$ is continuous and $\sum_{i=1}^{n} \delta^{i} \operatorname{sat}\left(h_{i}(z)\right)$ is bounded. Second, $\pi(z)$ is bounded from Lemma 2 . Third, the origin is an asymptotically stable equilibrium of the closed-loop of the state feedback control. Hence Assumption 2 in Atassi and Khalil (1999) is satisfied. Assumptions 1 and 3 for the system in Atassi and Khalil (1999) are also satisfied.

Take any compact set $C \in \mathbb{R}^{n}$ and $\hat{C} \in \mathbb{R}^{n}$ such that $z_{0} \in C$ and $\hat{z}_{0} \in \hat{C}$, then $1,2,3$ follow directly from Theorem 1 and Theorem 2 in Atassi and Khalil (1999). As to 4 , the finiteness of $\|y\|_{L^{2}\left(T_{\eta}\right)}$ is obtained from 2. Note that $\psi$ is continuous and $\hat{z}$ is bounded by 1 . Hence, $\|u\|_{L^{\infty}\left(\mathbb{R}_{+}\right)}$is also finite. So, $P\left(\Sigma\left(x_{0}\right)\right.$, $\left.\Xi_{H(\epsilon)}\left(\hat{z}_{0}\right)\right)$ is finite.

Now it is straightforward to uniformly bound the performance of system $\Sigma\left(x_{0}\right)$ for the Khalil design.

Theorem 2: Let $x_{0}$ be fixed and consider the system $\Sigma\left(x_{0}\right)$. Let $z_{0}=T\left(x_{0}\right)$. Let $\varphi_{i} \in C^{n-i}(\mathbb{R}), \varphi_{i}^{(k)} \in L^{\infty}(\mathbb{R})$, $1 \leq i \leq n ; 1 \leq k \leq n$. Then there is a positive constant $M$, such that for any $\tilde{z}_{0}$ there exists $\epsilon>0$ for which the controller $\Xi_{H(\epsilon)}\left(\hat{z}_{0}\right)$ achieves a uniformly bounded performance

$$
P\left(\Sigma\left(z_{0}\right), \Xi_{H(\epsilon)}\left(\hat{z}_{0}\right)\right)<M
$$

Proof: First note that

$$
\begin{aligned}
P\left(\Sigma\left(z_{0}\right), \Xi_{H(\epsilon)}\left(\hat{z}_{0}\right)\right) & =\int_{T_{\eta}}|y|^{2} \mathrm{~d} t+\|u\|_{L^{\infty}}\left(\mathbb{R}_{+}\right) \\
& =\int_{T_{\eta}}\left|z_{1}(t, \epsilon)\right|^{2} \mathrm{~d} t+\|u\|_{L^{\infty}}\left(\mathbb{R}_{+}\right)
\end{aligned}
$$

From Lemma 2, we know that $\psi(\hat{z})$ is bounded. So, the control input $u$ has a bound which is independent of $\hat{z}_{0}$. By Proposition 3, if $\epsilon$ is small enough, then $z_{1}(t, \epsilon)$ tends uniformly in $t$ to $\bar{z}_{1}(t)$, which is independent of $\hat{z}_{0}$ and uniformly bounded. Hence, $\bar{z}_{1}(t)$ has a bound that is independent of $\hat{z}_{0}$. Similarly the measure of the time set $T_{\eta}$ is also independent of $\hat{z}_{0}$ and finite. Hence the integral in (16) is finite and the bound is independent of $\hat{z}_{0}$. Therefore, we can find a constant $M$ such that (15) holds.

\subsection{Comparison}

Theorem 1 shows that for fixed initial state $x_{0}$, when the initial error $\left\|\tilde{x}_{0}\right\|$ becomes large, the performance of the $K K K$ design is not uniformly bounded even if $\varphi_{i}$ and its higher derivatives are globally bounded. On the other hand, Theorem 2 shows for the Khalil design, if $\varphi_{i}$ and its higher derivatives are globally bounded, then for any initial error $\tilde{z}_{0}$, through the high gain factor, we can design a globally bounded controller, achieving a uniformly bounded performance.

Hence we obtain the following comparative result.

Corollary 1: Consider the system $\Sigma\left(x_{0}\right)$, where $\varphi_{i} \in C^{n-i}(\mathbb{R}), \varphi_{i}^{(k)} \in L^{\infty}(\mathbb{R}), 1 \leq i \leq n$. Then there exist $\epsilon>0$ and $\hat{x}_{0}$ such that for any $\hat{z}_{0}$ we have

$$
P\left(\Sigma\left(z_{0}\right), \Xi_{H(\epsilon)}\left(\hat{z}_{0}\right)\right)<P\left(\Sigma\left(x_{0}\right), \Xi_{O}\left(\hat{x}_{0}\right)\right)
$$

Proof: The result follows directly from Theorems 1 and 2.

\section{Performance of parametric output feedback system}

In this section we study the performances of the $K K K$ design and the Khalil design for system

$$
\begin{aligned}
\Sigma\left(\theta, x_{0}\right): \quad \dot{x} & =A x+B(\theta \psi(y)+u), \quad x(0)=x_{0} \\
y & =C x
\end{aligned}
$$

where

$$
\begin{aligned}
x & =\left(x_{1}, x_{2}, \ldots, x_{n}\right)^{\mathrm{T}} \\
x_{0} & =\left(x_{01}, x_{02}, \ldots, x_{0 n}\right)^{\mathrm{T}}
\end{aligned}
$$

and $A, B$ and $C$ are as in $\S 2, \psi(y)$ is a Lipschitz continuous function and $\psi(0)=0$, and $\theta \in \mathbb{R}$ is an unknown constant. This is a parametric output feedback system, 
for which both $K K K$ and Khalil designs can be given to achieve regulation of the output and bounded performance.

To design a Khalil-type output feedback controller with a high gain observer, we need first to design a globally bounded state feedback controller. Generally, this is achieved by saturation of the state feedback controller. But we also require that the saturated controller stabilizes the system. For this purpose, we need to determine suitable saturation levels. However, the required saturation levels are typically dependent on $\theta$, the unknown constant. Therefore, we have to first quantify a priori estimates for the magnitude of $\theta$. Since $\theta$ is assumed to be unknown our knowledge of it is typically poor. Hence we have to estimate $\theta$ conservatively. But when our a priori upper bound for $|\theta|$ is conservative, we will show that the performance of the Khalil design becomes poor.

However, for a $K K K$ design, the performance is independent of the any a priori upper bound for $|\theta|$. Therefore, the performance keeps uniformly bounded as the a priori upper bound for $|\theta|$ becomes conservative.

Hence, for this system we will establish a result with the contrary performance relationship to that in $\S 2$.

\subsection{KKK design}

The $K K K$ design for the parametric output feedback system $\Sigma\left(\theta, x_{0}\right)$ as follows (Chapter 7 in Kristic et al. 1995).

Choose a vector $K$ such that $A_{0}=A-K C$ is Hurwitz, and define the filters

$$
\begin{aligned}
& \dot{\xi}_{0}=A_{0} \dot{\xi}_{0}+K y \\
& \dot{\xi}_{1}=A_{0} \xi_{1}+B \psi(y) \\
& \dot{v}_{0}=A_{0} v_{0}+e_{n} u
\end{aligned}
$$

The controller is defined by:

$$
\begin{aligned}
\Xi_{A}\left(\vartheta_{0}, \hat{x}_{0}\right): \quad u & =\alpha_{n} \\
\dot{\vartheta}_{1} & =\Gamma \omega_{1}\left(y, \bar{\xi}^{(2)}, \bar{v}^{(2)}\right) \zeta_{1} \\
\dot{\vartheta}_{2} & =\Gamma\left(\omega_{2}\left(y, \bar{\xi}^{(2)}, \bar{v}^{(2)}, \bar{\vartheta}^{(2)}\right)+\zeta_{1} e_{2}\right) \zeta_{2} \\
\dot{\vartheta}_{i} & =\Gamma \omega_{i}\left(y, \bar{\xi}^{(i)}, \bar{v}^{(i)}, \bar{\vartheta}^{(i-1)}\right) \zeta_{i}, \quad i=3, \ldots, n \\
\hat{x}(0) & =\hat{x}_{0}=\left(\hat{x}_{01}, \hat{x}_{02}, \ldots, \hat{x}_{0 n}\right)^{\mathrm{T}} \\
\vartheta(0) & =\vartheta_{0}=\left(\vartheta_{01}, \vartheta_{02}, \ldots, \vartheta_{0 n}\right)^{\mathrm{T}}
\end{aligned}
$$

where $e_{i}$ denotes the $i$ th coordinate vector in $\mathbb{R}^{2}$, and $\zeta_{i}, \omega_{i}, \alpha_{i}, i=1, \ldots, n$ are defined by the recursive expressions

$$
\begin{aligned}
& \zeta_{1}=y \\
& \zeta_{i}=v_{0, i}-\alpha_{i-1}\left(y, \bar{\vartheta}^{i}\right) \\
& \alpha_{1}=-\vartheta_{1}^{\mathrm{T}} \omega_{1} \\
& \alpha_{2}=-c_{2} \zeta_{2}-\vartheta_{2,2} \zeta_{1}-d_{2}\left(\frac{\partial \alpha_{1}}{\partial y}\right)^{2} \zeta_{2}+\frac{\partial \alpha_{1}}{\partial y} \xi_{0,2} \\
& -\vartheta_{2}^{\mathrm{T}} \omega_{2}+k_{2} v_{0,1}+\frac{\partial \alpha_{1}}{\partial \xi_{0}}\left(A_{0} \xi_{0}+K y\right) \\
& +\frac{\partial \alpha_{1}}{\partial \xi_{1}}\left(A_{0} \xi_{1}+B \psi(y)\right)+\frac{\partial \alpha_{1}}{\partial v_{0}} A_{0} v_{0}+\frac{\partial \alpha_{1}}{\partial \vartheta_{1}} \Gamma \omega_{1} \zeta_{1} \\
& \alpha_{i}=-c_{i} \zeta_{i}-d_{i}\left(\frac{\partial \alpha_{i-1}}{\partial y}\right)^{2} \zeta_{i}+\frac{\partial \alpha_{i-1}}{\partial y} \xi_{0,2}-\vartheta_{i}^{\mathrm{T}} \omega_{i}+k_{i} v_{0,1} \\
& +\frac{\partial \alpha_{i-1}}{\partial \xi_{0}}\left(A_{0} \xi_{0}+K y\right)+\frac{\partial \alpha_{i-1}}{\partial \xi_{1}}\left(A_{0} \xi_{1}+B \psi(y)\right) \\
& +\frac{\partial \alpha_{i-1}}{\partial v_{0}} A_{0} v_{0}+\frac{\partial \alpha_{i-1}}{\partial \vartheta_{1}} \Gamma \omega_{1} \zeta_{1}+\frac{\partial \alpha_{i-1}}{\partial \vartheta_{2}} \Gamma\left(\omega_{2}+\zeta_{1} e_{2}\right) \zeta_{2} \\
& +\sum_{j=3}^{i-1} \frac{\partial \alpha_{i-1}}{\partial \vartheta_{j}} \Gamma \omega_{j} \zeta_{j}, \quad i=3, \ldots, n \\
& \omega_{1}^{\mathrm{T}}=\left(c_{1} \zeta_{1}+d_{1} \zeta_{1}+\xi_{0,2}, v_{0,2}\right) \\
& \omega_{i}^{\mathrm{T}}=-\frac{\partial \alpha_{i-1}}{\partial y}\left(\xi_{1,2}, v_{0,2}\right), \quad i=2, \ldots, n-1 \\
& \omega_{n}^{\mathrm{T}}=-\frac{\partial \alpha_{n-1}}{\partial y}\left(\varphi+\xi_{1,2}, v_{0,2}\right) \\
& \bar{\xi}^{(i)}=\left(\xi_{0,1}, \ldots, \xi_{0, i}, \ldots, \xi_{1,1}, \ldots, \xi_{1, i}\right), \quad i=1, \ldots, n \\
& \bar{v}^{(i)}=\left(v_{0,1}, \ldots, v_{0, i}\right), \quad i=1, \ldots, n \\
& \bar{\vartheta}_{(i)}=\left(\vartheta_{1}^{\mathrm{T}}, \ldots, \vartheta_{i}^{\mathrm{T}}\right), \quad i=1, \ldots, n
\end{aligned}
$$

We summarize the relevant well-known properties of this controller in the following proposition.

Proposition 4: For the system $\Sigma\left(\theta, x_{0}\right)$ the controller $\Xi_{A}\left(\vartheta_{0}, \hat{x}_{0}\right)$ guarantees the global boundedness of all signals and the regulation of output

$$
\lim _{t \rightarrow \infty} y(t)=0
$$

Moreover, the controller achieves bounded performance

$$
P\left(\Sigma\left(\theta, x_{0}\right), \Xi_{A}\left(\vartheta_{0}, \hat{x}_{0}\right)\right)<\infty
$$

\subsection{Khalil design}

We define a Khalil controller using the non-linear separation principle (Atassi and Khalil 1999). The standard steps in this synthesis procedure is as follows: firstly design a state feedback controller; then saturate 
the controller out off some sets based on our a priori knowledge of the worst case bounds for the closed loop signals; next replace the unmeasurable state variables by the estimated states from a high gain observer. This defines an output feedback control.

3.2.1. Controller design. Firstly we design a state feedback controller based on Lyapunov theory, and obtain a priori worst case estimates for the bounds of the closed loop signals.

We chose a vector

$$
K=\left(k_{1}, k_{2}, \ldots, k_{n}\right)
$$

such that matrix $A+B K$ is Hurwitz, and let matrix $P$ be the positive definite symmetric solution of the Lyapunov equation

$$
(A+B K)^{\mathrm{T}} P+P(A+B K)=-I
$$

By considering the Lyapunov function

$$
V(x, \hat{\theta})=x^{\mathrm{T}} P x+\frac{1}{2}(\theta-\hat{\theta})^{2}
$$

we define a state feedback controller

$$
\begin{aligned}
\Xi_{s}\left(\hat{\theta}_{0}, x_{0}\right): \quad u & =\mu(x, \hat{\theta})=K x-\hat{\theta} \psi(y) \\
\dot{\hat{\theta}} & =v(x, \hat{\theta})=2 x^{\mathrm{T}} P B \psi(y), \quad \hat{\theta}(0)=\hat{\theta}_{0}
\end{aligned}
$$

noting that along the solution of the closed loop, we have

$$
\dot{V}=-x^{\mathrm{T}} x \leq 0
$$

This suffices to show global stability and regulation of the output to zero.

To design an output feedback controller through a high gain observer, the functions $\mu$ and $v$ should be globally bounded (Atassi and Khalil 1999). So, we saturate $\mu$ and $v$ outside some suitably defined sets which ensure that the modified controller still stabilizes the system. For this purpose, we utilize a priori estimates of $x$ and $\hat{\theta}$.

Firstly, from $\dot{V} \leq 0$, we get

$$
\frac{1}{2}(\theta-\hat{\theta})^{2} \leq V(t) \leq V(0)=x_{0}^{\mathrm{T}} P x_{0}+\frac{1}{2}\left(\theta-\hat{\theta}_{0}\right)^{2}
$$

Hence

$$
|\hat{\theta}| \leq \theta_{m}+\sqrt{2 \bar{\lambda}(P) \chi_{m}^{2}+\left(\theta_{m}+\left|\hat{\theta}_{0}\right|\right)^{2}}=: \Theta_{0}
$$

where $\theta_{m}$ and $\chi_{m}$ are the a priori estimates of upper bound for the magnitude of the unknown parameter $\theta$ and the magnitude of the initial state $\left|x_{0}\right|$, and $\bar{\lambda}(P)$ is the largest eigenvalue of $P$.
Similarly,

$$
\begin{aligned}
\|x\|=\left(x^{\mathrm{T}} x\right)^{1 / 2} & \leq\left(\frac{1}{\underline{\lambda}(P)} x^{\mathrm{T}} P x\right)^{1 / 2} \leq\left(\frac{1}{\underline{\lambda}(P)} V(0)\right)^{1 / 2} \\
& \leq\left(\frac{1}{\underline{\lambda}(P)}\left(\bar{\lambda}(P) \chi_{m}^{2}+\frac{1}{2}\left(\theta_{m}+\left|\hat{\theta}_{0}\right|\right)^{2}\right)\right)^{1 / 2} \\
& =: X_{0}
\end{aligned}
$$

where $\underline{\lambda}(P)$ is the smallest eigenvalue of $P$ and

$$
|y|=\left|x_{1}\right| \leq\|x\| \leq X_{0}
$$

Finally, from (18a)

$$
|\mu| \leq n \bar{k} X_{0}+\Theta_{0} \Psi_{0}=: U_{0}
$$

where

$$
\begin{aligned}
\bar{k} & =\max _{1 \leq j \leq n}\left\{\left|k_{j}\right|\right\} \\
\Psi_{0} & =\sup _{\left|x_{1}\right| \leq X_{0}}\left\{\left|\psi\left(x_{1}\right)\right|\right\}
\end{aligned}
$$

On the other hand, suppose that $p$ is the biggest element in the last row of $P$, then by $(18 b)$ we obtain

$$
|\nu| \leq n p\|x\| \Psi_{0} \leq n p X_{0} \Psi_{0}=: V_{0}
$$

Now we saturate $\mu$ and $v$ as

$$
\begin{aligned}
& \mu_{s}(x, \hat{\theta})=U_{0} \operatorname{sat}\left(\frac{\mu(x, \hat{\theta})}{U_{0}}\right) \\
& v_{s}(x, \hat{\theta})=V_{0} \operatorname{sat}\left(\frac{\nu(x, \hat{\theta})}{V_{0}}\right)
\end{aligned}
$$

to obtain a globally bounded state feedback controller

$$
\begin{aligned}
\Xi_{s}^{b}\left(\theta_{m}, \chi_{m}, \hat{\theta}_{0}, x_{0}\right): & u=\mu_{s}(x, \hat{\theta}) \\
\dot{\hat{\theta}} & =v_{s}(x, \hat{\theta}), \quad \hat{\theta}(0)=\hat{\theta}_{0}
\end{aligned}
$$

Consequently a Khalil controller can be obtained as

$$
\begin{aligned}
& \Xi_{H(\epsilon)}\left(\theta_{m}, \chi_{m}, \hat{\theta}_{0}, \hat{x}_{0}\right): u=\mu_{s}(\hat{x}, \hat{\theta}) \\
& \dot{\hat{\theta}}=v_{s}(\hat{x}, \hat{\theta}), \quad \hat{\theta}(0)=\hat{\theta}_{0} \quad(25 b) \\
& \dot{\hat{x}}=A \hat{x}+H\left(y-\hat{x}_{1}\right), \quad \hat{x}(0)=\hat{x}_{0}
\end{aligned}
$$

The properties of this controller are summarized in the following Proposition.

Proposition 5: For the system $\Sigma\left(\theta, x_{0}\right)$, if $|\theta| \leq \theta_{m}$, then when $\epsilon$ is small enough, the controller $\Xi_{H(\epsilon)}\left(\theta_{m}, \chi_{m}\right.$, $\left.\hat{\theta}_{0}, \hat{x}_{0}\right)$ guarantees the global boundedness of all signals and the regulation of output

$$
\lim _{t \rightarrow \infty} y(t)=0
$$


Moreover, the controller achieves bounded performance

$$
P\left(\Sigma\left(\theta, x_{0}\right), \Xi_{H(\epsilon)}\left(\theta_{m}, \chi_{m}, \hat{\theta}_{0}, \hat{x}_{0}\right)\right)<\infty
$$

Proof: The system $\Sigma\left(\theta, x_{0}\right)$ is global normal form, hence satisfies Assumptions 1 and 2 in Khalil (1996). The state feedback control input $\mu_{s}(x, \hat{\theta})$ and update law $v_{s}(x, \hat{\theta})$ are defined the same as those in Khalil (1996). So, by Theorem 2 of Khalil (1996), we obtain the boundedness of all signals and the regulation of output.

The proof of the boundedness of the performance follows from the boundedness of the closed-loop signals.

3.2.2. Performance. First we are going to establish the following lemma.

Lemma 3: Let

$$
e_{0 j}=x_{0 j}-\hat{x}_{0 j}, \quad 1 \leq j \leq n
$$

and suppose that at least one of $e_{0 j}, 1 \leq j \leq n-1$, is not equal to zero. Then for the closed loop $\left(\Sigma\left(\theta, x_{0}\right)\right.$, $\left.\Xi_{H(\epsilon)}\left(\theta_{m}, \chi_{m}, \hat{\theta}_{0}, \hat{x}_{0}\right)\right)$, we have

$$
\lim _{\epsilon \rightarrow 0}\|u\|_{L^{\infty}\left(\mathbb{R}^{+}\right)}=U_{0}
$$

Proof: From the definition of the controller $\Xi_{H(\epsilon)}\left(\theta_{m}\right.$, $\left.\chi_{m}, \hat{\theta}_{0}, \hat{x}_{0}\right)$, it suffices to prove that

$$
\lim _{\epsilon \rightarrow 0}\left(\sup _{t \in \mathbb{R}^{+}}\|\hat{x}(t)\|\right)=\infty
$$

Now let

$$
\begin{array}{ll}
e_{j}=x_{j}-\hat{x}_{j}, & 1 \leq j \leq n \\
\zeta_{j}=\frac{1}{\epsilon^{n-j}} e_{j}, & 1 \leq j \leq n
\end{array}
$$

Then the closed loop $\left(\Sigma\left(\theta, x_{0}\right), \Xi_{H(\epsilon)}\left(\theta_{m}, \chi_{m}, \hat{\theta}_{0}, \hat{x}_{0}\right)\right)$ is given by

$$
\begin{gathered}
\dot{x}=A x+B\left(\hat{\theta} \psi(y)+\mu_{s}(\hat{x}, \hat{\theta})\right), \quad x(0)=x_{0} \\
\dot{\hat{\theta}}=v_{s}(\hat{x}, \hat{\theta}), \quad \hat{\theta}(0)=\hat{\theta}_{0} \\
\epsilon \dot{\zeta}=D \zeta+\epsilon B\left(\hat{\theta} \psi(y)+\mu^{s}(\hat{x}, \hat{\theta})\right), \quad \zeta(0)=\zeta_{0}
\end{gathered}
$$

where $L=\left(\alpha_{1}, \alpha_{2}, \ldots, \alpha_{n}\right)^{\mathrm{T}}$, the matrix $D=A-L C$ is Hurwitz and

$$
\zeta_{0}=\left(\frac{e_{01}}{\epsilon^{n-1}}, \ldots, \frac{e_{0, n-1}}{\epsilon}, e_{0 n}\right)^{\mathrm{T}}
$$

To prove (27) it is enough to show

$$
\lim _{\epsilon \rightarrow 0}\left(\sup _{t \in \mathbb{R}^{+}}\|e(t)\|\right)=\infty
$$

Since $\xi_{n}=e_{n}$, it is sufficient to show

$$
\lim _{\epsilon \rightarrow 0}\left(\sup _{t \in \mathbb{R}^{+}}\left|\zeta_{n}(t)\right|\right)=\infty
$$

On the other hand, let $t=\tau \epsilon$, then $(28 c)$ can be written into

$$
\frac{\mathrm{d} \zeta}{\mathrm{d} \tau}=D \zeta+\epsilon B\left(\hat{\theta} \psi(y)+\mu^{s}(\hat{x}, \hat{\theta})\right), \quad \zeta(0)=\zeta_{0}
$$

When $\epsilon$ is small enough, the output $y=x_{1}$ converges uniformly in $t$ to the solution of state feedback closed system, and hence is uniformly bounded, therefore $\psi(y)$ is uniformly bounded. So, the term $B(\hat{\theta} \psi(y)+$ $\left.\mu^{s}(\hat{x}, \hat{\theta})\right)$ in (31) is bounded uniformly in $\tau$. Therefore, when $\epsilon \rightarrow 0$, the solution of (31) is convergent uniformly in $\tau$ to the solution of

$$
\frac{\mathrm{d} \eta}{\mathrm{d} \tau}=D \eta, \quad \eta(0)=\zeta_{0}
$$

Hence, we only need to show

$$
\lim _{\epsilon \rightarrow 0}\left(\sup _{t \in \mathbb{R}^{+}}\left|\eta_{n}(t)\right|\right)=\infty
$$

Note that

$$
D=\left(\begin{array}{ccccc}
-d_{1} & 1 & 0 & \cdots & 0 \\
-d_{2} & 0 & 1 & \cdots & 0 \\
-d_{n-1} & 0 & 0 & \cdots & 1 \\
-d_{n} & 0 & 0 & \cdots & 0
\end{array}\right)
$$

and by induction we can show

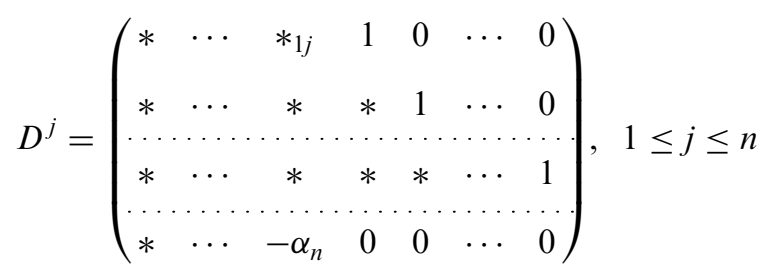

where the $*$ s are elements which do not need to be specified. Let

$$
s=\min _{1 \leq j \leq n-1}\left\{j \mid e_{0 j} \neq 0\right\}
$$

and consider the time

$$
t_{\epsilon}=\epsilon \gamma_{\epsilon}
$$

where

$$
\gamma_{\epsilon}=\epsilon^{(n-s-\beta) / s}
$$

and

$$
\beta=\frac{1}{2} \min \left\{\frac{n-s}{s+1}, 1\right\}
$$


Note the solution of (32) is given by

$$
\eta(\tau)=\mathrm{e}^{D \tau} \zeta_{0}
$$

i.e. equivalently by

$$
\eta(t)=\mathrm{e}^{D(t / \epsilon)} \zeta_{0}
$$

Hence

$$
\eta\left(t_{\epsilon}\right)=\mathrm{e}^{D \gamma_{\epsilon}} \zeta_{0}
$$

\subsection{Comparison}

For the system $\Sigma\left(\theta, x_{0}\right)$, as the a priori estimate of upper bound $\theta_{m}$ for the uncertain parameter $\theta$ becomes conservative, Proposition 4 shows that the $K K K$ design guarantees uniform bounded performance of the controller, whereas Theorem 3 shows that the performance of the Khalil design becomes large. Here we have the following comparative result.

Now

$$
\begin{aligned}
& \mathrm{e}^{D \gamma_{\epsilon}}=I+\gamma_{\epsilon} D+\frac{\gamma_{\epsilon}^{2}}{2 !} D^{2}+\cdots+\frac{\gamma_{\epsilon}^{s}}{s !} D^{s}+o\left(\overline{\gamma_{\epsilon}^{s+(1 / 2)}}\right)
\end{aligned}
$$

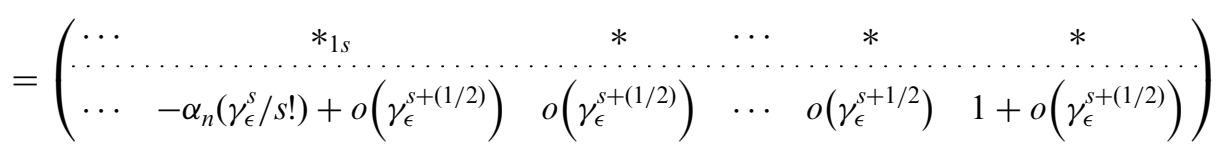

Noting that $e_{0 j}=0$ for $1 \leq j \leq s-1$, yields

$$
\begin{aligned}
\eta_{n}\left(t_{\epsilon}\right)= & \left(-\alpha_{n} \frac{\gamma_{\epsilon}^{s}}{s !}+o\left(\gamma_{\epsilon}^{s+(1 / 2)}\right)\right) \frac{e_{0 s}}{\epsilon^{n-s}}+o\left(\gamma_{\epsilon}^{s+(1 / 2)}\right) \frac{e_{0, s+1}}{\epsilon^{n-s-1}} \\
& +\cdots+o\left(\gamma_{\epsilon}^{s+(1 / 2)}\right) \frac{e_{0, n-1}}{\epsilon}+\left(1+o\left(\gamma_{\epsilon}^{s+(1 / 2)}\right)\right) e_{0 n} \\
= & -\frac{\alpha_{n} e_{0 s}}{s ! \epsilon^{\beta}}+e_{0 n}+o\left(\epsilon^{\lambda}\right)
\end{aligned}
$$

where

$$
\lambda=\frac{n-s+\beta}{2 s}>0
$$

But by assumption, $\alpha_{n}>0$, and $e_{0 s} \neq 0$. Therefore, (36) implies (33). This completes the proof.

From this lemma, we can obtain the following theorem.

Theorem 3: Let $\theta, x_{0}$ be fixed, and suppose $\left|x_{0}\right| \leq \chi_{m}$. Consider the system $\Sigma\left(\theta, x_{0}\right)$ and the controller $\Xi_{H(\epsilon)}\left(\theta_{m}, \chi_{m}, \hat{\theta}_{0}, \hat{x}_{0}\right)$. Suppose that at least one of the initial errors $e_{0 j}, 1 \leq j \leq n-1$, is not equal to zero. Then for the closed loop system $\left(\Sigma\left(\theta, x_{0}\right), \Xi_{H(\epsilon)}\left(\theta_{m}, \chi_{m}, \hat{\theta}_{0}, \hat{x}_{0}\right)\right)$, we have

$$
\lim _{\epsilon \rightarrow 0}\left(\lim _{\theta_{m} \rightarrow \infty} P\left(\Sigma\left(\theta, x_{0}\right), \Xi_{H(\epsilon)}\left(\theta_{m}, \chi_{m}, \hat{\theta}_{0}, \hat{x}_{0}\right)\right)\right)=\infty
$$

Proof: For the closed loop system $\left(\Sigma\left(\theta, x_{0}\right)\right.$, $\left.\Xi_{H(\epsilon)}\left(\theta_{m}, \chi_{m}, \hat{\theta}_{0}, \hat{x}_{0}\right)\right)$, the saturation levels $U_{0}$ and $V_{0}$ for the output feedback controller are dependent on $\theta_{m}$, the a priori estimate of upper bound for the unknown parameter $\theta$. When $\theta_{m}$ is large, from (19)-(22), $U_{0}$ and $V_{0}$ are large. By Lemma 3, as the high gain factor $\epsilon$ is small, $\|u\|_{L^{\infty}\left(\mathbb{R}^{+}\right)}$is also large, that is the performance becomes large.
Corollary 2: Let $\theta, x_{0}$ be fixed, and suppose $\left|x_{0}\right| \leq \chi_{m}$. For the system $\Sigma\left(\theta, x_{0}\right)$, if the bound $\theta_{m}$ for the unknown parameter $\theta$ is conservative enough, and the gain factor $\epsilon$ is small enough, then

$$
P\left(\Sigma\left(\theta, x_{0}\right), \Xi_{A}\left(\vartheta_{0}, \hat{x}_{0}\right)\right)<P\left(\Sigma\left(\theta, x_{0}\right), \Xi_{H(\epsilon)}\left(\theta_{m}, \chi_{m}, \hat{\theta}_{0}, \hat{x}_{0}\right)\right)
$$

Proof: The result follows directly from Proposition 4 and Theorem 3.

\section{Conclusion}

Through the comparison of performances for $K K K$ and Khalil designs, we have established the following results:

- For output feedback system, the performance of $K K K$ design is sensitive to the initial datum of the observer. The performance of the $K K K$ design is not uniformly bounded in the initial error between the initial datum of the state and the initial datum of the observer. When the initial error becomes large, the performance becomes large. Whereas, for the Khalil design, for any initial error, by choosing small high gain factor, we can design a globally bounded controller, achieving a uniformly bounded performance. Therefore, if the initial error is large or in the case that we have poor information for the initial datum of the state, the Khalil design has better performance than the KKK design.

- For parametric output feedback system, the performance of the $K K K$ design is independent of the a priori estimate bound of the uncertain parameter. When the a priori estimate becomes conservative the performance remains uniformly bounded. Whilst, for the Khalil design, the performance is dependent on the saturation levels for the 
controller and the adaptive law, that is dependent on the a priori estimate bound of the uncertain parameter, and the performance becomes large as the a priori estimate becomes conservative. Hence, if we have poor information for the unknown parameter and the a priori estimate bound is conservative, the $K K K$ design has better performance than the Khalil design.

The primary contribution of this paper is to provide rigorous statements and proofs of the intuitively reasonable trade-offs in performance between the differing classes of designs. The results have been expressed in qualitative terms only. The purpose of the paper is to illustrate the asymptotic differences between the designs. It should also be noted that the results are asymptotic in nature, that is they require some parameter (either an initial condition or an uncertainty level) to be large in order to make the required comparison. Of course, in practice these parameters cannot be arbitrarily large without causing the control to run into physical limits. A more quantitative approach is challenging, as achieving tight bounds on non-singular performance is difficult. This is an interesting avenue for future research.

\section{Acknowledgement}

The authors are grateful to the reviewers for their helpful comments and constructive suggestions.

\section{Appendix. The proof of proposition 1}

Consider the closed loop $\left(\Sigma^{0}\left(x_{0}\right), \Xi_{O}^{0}\left(x_{0}\right)\right)$. First, observe that the observation error $\tilde{x}=x-\hat{x}$ satisfies

$$
\begin{aligned}
& \dot{\tilde{x}}_{1}=-k_{1} \tilde{x}_{1}+\tilde{x}_{2} \\
& \dot{\tilde{x}}_{2}=-k_{2} \tilde{x}_{1}, \quad \tilde{x}(0)=\tilde{x}_{0}
\end{aligned}
$$

hence satisfies equation

$$
\begin{aligned}
& \ddot{\tilde{x}}_{1}+k_{1} \dot{\tilde{x}}_{1}+k_{2} \tilde{x}_{1}=0 \\
& \tilde{x}_{1}(0)=x_{01}-\hat{x}_{01} \\
& \dot{\tilde{x}}_{1}(0)=x_{02}-\hat{x}_{02}-k_{1}\left(x_{01}-\hat{x}_{01}\right)
\end{aligned}
$$

where

$$
\tilde{x}_{0}=x_{0}-\hat{x}_{0}=\left(x_{01}-\hat{x}_{01}, x_{02}-\hat{x}_{02}\right)^{\mathrm{T}}
$$

Second, note that the control signal $u$ can be expressed as

$$
\Xi_{O}^{0}\left(\hat{x}_{0}\right): \quad u=\alpha_{2}\left(y, \hat{x}_{1}, \hat{x}_{2}\right)=k_{2} \hat{x}_{1}-h_{2} \hat{x}_{2}-h y-\varphi(y)
$$

where

$$
\begin{gathered}
h=\left(c_{2}+d_{2}\left(c_{1}+d_{1}\right)^{2}\right)\left(c_{1}+d_{1}\right)+k_{2}+1 \\
h_{2}=c_{2}+d_{2}\left(c_{1}+d_{1}\right)^{2}+c_{1}+d_{1}
\end{gathered}
$$

So, the closed loop system can be written as

$$
\begin{aligned}
& \dot{x}_{1}=x_{2} \\
& \dot{x}_{2}=-h x_{1}+k_{2} \hat{x}_{1}-h_{2} \hat{x}_{2} \\
& \dot{\hat{x}}_{1}=k_{1} x_{1}-k_{1} \hat{x}_{1}+\hat{x}_{2} \\
& \dot{\hat{x}}_{2}=-h_{1} x_{1}-h_{2} \hat{x}_{2}
\end{aligned}
$$

where

$$
h_{1}=h-k_{2}=\left(c_{2}+d_{2}\left(c_{1}+d_{1}\right)^{2}\right)\left(c_{1}+d_{1}\right)+1
$$

Consider the first situation $\hat{x}_{0}=x_{0}$, namely, $\tilde{x}_{0}=0$. The solution of (37) is $\tilde{x}=0$, so $\hat{x}(t) \equiv x(t)$, and the closed system (40) reduces to

$$
\begin{aligned}
& \dot{x}_{1}=x_{2} \\
& \dot{x}_{2}=-h_{1} x_{1}-h_{2} x_{2}
\end{aligned}
$$

Thus we have

$$
\begin{aligned}
& \ddot{x}_{1}+h_{2} \dot{x}_{1}+h_{1} x_{1}=0 \\
& x_{1}(0)=x_{01}, \quad \dot{x}_{1}(0)=x_{02}
\end{aligned}
$$

Write the solution of the above equation as $x_{1}^{0}(t)$, and observe that $x_{1}^{0}(t)$ can be expressed as

$$
x_{1}^{0}(t)=x_{01} q_{1}(t)+x_{02} q_{2}(t)
$$

where $q_{1}, q_{2}$ are functions which are independent of $x_{01}, x_{02}$. Moreover, we can choose $\uparrow c_{i}, d_{i}, i=1,2$ such that $q_{2}(t)>0$ for $t>0$, and further $x_{1}^{0}(t)>0$ for $t>0$ if $x_{02}>0$.

Now consider the second situation $\hat{x}_{01}=x_{01}$ and $\hat{x}_{02}=0$, namely, $\tilde{x}_{01}=0$ and $\tilde{x}_{02}=x_{02}$. So, the problem (38) becomes

$$
\begin{aligned}
& \ddot{\tilde{x}}_{1}+k_{1} \dot{\tilde{x}}_{1}+k_{2} \tilde{x}_{1}=0 \\
& \tilde{x}_{1}(0)=0, \quad \dot{\tilde{x}}_{1}(0)=x_{02}
\end{aligned}
$$

The solution of the above problem can be written as

$$
\tilde{x}_{1}=x_{02} f_{1}(t)
$$

$\dagger$ For example, we can choose $c_{i}, d_{i}, i=1,2$ such that $h_{2}^{2}>4 h_{1}$, and let

$$
\lambda_{1}=-\frac{1}{2}\left(h_{2}-\sqrt{h_{2}^{2}-4 h_{1}}\right), \quad \lambda_{2}=-\frac{1}{2}\left(h_{2}+\sqrt{h_{2}^{2}-4 h_{1}}\right)
$$

then $q_{1}(t)$ and $q_{2}(t)$ can be written as

$$
\begin{aligned}
& q_{1}(t)=\frac{1}{\lambda_{1}-\lambda_{2}}\left(\lambda_{1} \mathrm{e}^{\lambda_{2} t}-\lambda_{2} \mathrm{e}^{\lambda_{1} t}\right), \\
& q_{2}(t)=\frac{1}{\lambda_{1}-\lambda_{2}}\left(\mathrm{e}^{\lambda_{1} t}-\mathrm{e}^{\lambda_{2} t}\right)>0, \quad t>0
\end{aligned}
$$

$\$$ Here, $x_{1}^{0}(t)$ can also be written as

$$
x_{1}^{0}(t)=\frac{1}{\lambda_{1}-\lambda_{2}}\left(\left(x_{02}-\lambda_{1} x_{01}\right) \mathrm{e}^{\lambda_{1} t}-\left(x_{02}-\lambda_{2} x_{01}\right) \mathrm{e}^{\lambda_{2} t}\right)
$$

It can verify that if $x_{02}>0$ then $\dot{x}_{1}^{0}(t)>0$ for $t>0$. Note that $x_{1}^{0}(0)=0$, then $x_{1}^{0}(t)>0$ for $t>0$. 
where $f_{1}(t)$ is a continuous function which is independent of $x_{02}$. At the same time, $\tilde{x}_{2}$ can be written as

$$
\tilde{x}_{2}=x_{02} f_{2}(t)
$$

also where $f_{2}(t)$ is a continuous function which is independent of $x_{02}$.

Now substitute $\hat{x}=x-\tilde{x}$ into the closed loop (40), and rewrite the first two equations as

$$
\begin{aligned}
& \dot{x}_{1}=x_{2} \\
& \dot{x}_{2}=-h_{1} x_{1}-h_{2} x_{2}-k_{2} \tilde{x}_{1}+h_{2} \tilde{x}_{2}
\end{aligned}
$$

or

$$
\ddot{x}_{1}+h_{2} \dot{x}_{1}+h_{1} x_{1}=x_{02} f(t)
$$

where

$$
f(t)=-k_{2} f_{1}(t)+h_{2} f_{2}(t)
$$

is also independent of $x_{02}$. Again we can choose $k_{1}, k_{2}$ such that $f(t)>0$.

Solve the following problem

$$
\begin{aligned}
& \ddot{x}_{1}+h_{2} \dot{x}_{1}+h_{1} x_{1}=x_{02} f(t) \\
& x_{1}(0)=x_{01}, \quad \dot{x}_{1}(0)=x_{02}
\end{aligned}
$$

We can express the solution of (48) as

$$
x_{1}(t)=x_{1}^{0}(t)+\int_{0}^{t} \phi(t-\tau) x_{02} f(\tau) \mathrm{d} \tau
$$

where $\phi(t)$ is the solution of $(42 a)$ which satisfies $\phi(0)=0$ and $\dot{\phi}(0)=1$, namely $\phi(t)=q_{2}(t)$. Write

$$
g(t)=\int_{0}^{t} q_{2}(t-\tau) f(\tau) \mathrm{d} \tau
$$

then

$$
x_{1}(t)=x_{1}^{0}(t)+x_{02} g(t)
$$

where $g(t)>0$.

Writing

$$
\begin{aligned}
& T_{\eta}=\left\{t \geq 0|| x_{1}(t) \mid>\eta\right\} \\
& T_{\eta}^{0}=\left\{t \geq 0|| x_{1}^{0}(t) \mid>\eta\right\}
\end{aligned}
$$

$\S$ We choose $k_{2}^{2}>4 k_{2}$, and let

$$
\mu_{1}=-\frac{1}{2}\left(k_{1}-\sqrt{k_{1}^{2}-4 k_{2}}\right), \quad \mu_{2}=-\frac{1}{2}\left(k_{1}+\sqrt{k_{1}^{2}-4 k_{2}}\right)
$$

then we get

$$
\begin{aligned}
f_{1}(t) & =\frac{1}{\mu_{1}-\mu_{2}}\left(\mathrm{e}^{\mu_{1} t}-\mathrm{e}^{\mu_{2} t}\right), \quad f_{2}(t)=\frac{1}{\mu_{1}-\mu_{2}}\left(-\mu_{2} \mathrm{e}^{\mu_{1} t}+\mu_{1} \mathrm{e}^{\mu_{2} t}\right) \\
f(t) & =\frac{1}{\mu_{1}-\mu_{2}}\left(\left(-h_{2} \mu_{2}-k_{2}\right) \mathrm{e}^{\mu_{1} t}+\left(h_{2} \mu_{1}+k_{2}\right) \mathrm{e}^{\mu_{2} t}\right)
\end{aligned}
$$

So, if $h_{2} k_{1}>2 k_{2}$ we obtain $f(t)>0$. then $T_{\eta}^{0} \subset T_{\eta}$ since $x_{1}(t)>x_{1}^{0}(t)$. Hence

$$
\begin{aligned}
& \left\|x_{1}\right\|_{L^{2}\left(T_{\eta}\right)}^{2}-\left\|x_{1}^{0}\right\|_{L^{2}\left(T_{\eta}^{0}\right)}^{2} \\
& \geq \int_{T_{\eta}^{0}}\left(\left(x_{1}(t)\right)^{2}-\left(x_{1}^{0}(t)\right)^{2}\right) \mathrm{d} t \\
& =x_{02}^{2} a+x_{02} b
\end{aligned}
$$

where

$$
a=\int_{T_{\eta}^{0}}\left(g(t)^{2}+2 q_{2}(t) g(t)\right) \mathrm{d} t
$$

is a positive constant since $g(t), q_{2}(t)>0$ and

$$
b=\int_{T_{\eta}^{0}} 2 x_{01} q_{1}(t) \mathrm{d} t
$$

is a constant which is independent of $x_{02}$.

Write the control input of controller $\Xi_{O}^{0}\left(x_{01}, x_{02}\right)$ as $u^{0}$, and the control input of controller $\Xi_{O}^{0}\left(x_{01}, 0\right)$ as $u^{1}$. Then by a tedious calculation, we can obtain

$$
\begin{aligned}
\left\|u^{0}\right\| & \leq x_{02} a_{0}+b_{0} \\
\left\|u^{1}\right\| & \leq x_{02} a_{1}+b_{1}
\end{aligned}
$$

since $\varphi$ is Lipschitz continuous, where $a_{i}, b_{i}, i=1,2$, are positive constants which are independent of $x_{02}$. Therefore, from (49) and (50), we obtain

$$
\begin{aligned}
& \lim _{x_{02} \rightarrow \infty} \frac{1}{x_{02}^{2}} P\left(\Sigma^{0}\left(x_{0}\right), \Xi_{O}^{0}\left(x_{01}, 0\right)\right)-P\left(\Sigma^{0}\left(x_{0}\right), \Xi_{O}^{0}\left(x_{01}, x_{02}\right)\right) \\
& =\lim _{x_{02} \rightarrow \infty} \frac{1}{x_{02}^{2}}\left(\left\|x_{1}\right\|_{L^{2}\left(T_{\eta}\right)}^{2}-\left\|x_{1}^{0}\right\|_{L^{2}\left(T_{\eta}^{0}\right)}^{2}\right) \\
& \quad+\frac{1}{x_{02}^{2}}\left(\left\|u^{1}\right\|-\left\|u^{0}\right\| x_{02}^{2}\right) \geq a>0
\end{aligned}
$$

So, finally, we obtain that

$$
\begin{aligned}
& \lim _{x_{02} \rightarrow \infty}\left(P\left(\Sigma^{0}\left(x_{0}\right), \Xi_{O}^{0}\left(x_{01}, 0\right)\right)\right. \\
& \quad-P\left(\Sigma^{0}\left(x_{0}\right), \Xi_{O}^{0}\left(x_{01}, x_{02}\right)\right)=+\infty
\end{aligned}
$$

This completes the proof.

\section{References}

Atassi, A., and Khalil, H., 1999, A separation principle for the stabilization of a class of nonlinear systems. IEEE Transactions on Automatic Control, 44, 1672-1687.

Beleznay, F., and French, M., 2003, Overparameterised adaptive controllers can reduce nonsingular costs. Systems and Control Letters, 48, 12-25.

EsFandiari, F., and Khalil, H., 1992, Output feedback stabilization of fully linearizable systems. International Journal of Control, 56, 1007-1037.

French, M., Szepesvári, Cs., and Rogers, E., 2000, Uncertainty, performance, and model dependency in approximate adaptive nonlinear control. IEEE Transactions on Automatic Control, 45, 353-358.

FRENCH, M., 2002, An analytical comparison between the nonsingular quadratic performance of robust and adaptive 
backstepping designs. IEEE Transactions on Automatic Control, 47, 670-675.

IsIDORI, A., 1989, Nonlinear Control Systems (New York: Springer-Verlag) 2nd edition.

Khalil, H., and Esfandiari, F., 1993, Semiglobal stabilization of a class of nonlinear systems using output feedback. IEEE Transactions on Automatic Control, 38, 1412-1415.

KHaliL, H., 1996, Adaptive output feedback control of nonlinear systems represented by input-output models. IEEE Transactions on Automatic Control, 41, 177-188.
Khalil, H., 1999, Comparison of different techniques for nonlinear output feedback adaptive control. Proceeding of the 38th Conference on Decision and Control, Phoenix, AZ, USA, December.

Kristic, M., Kanellakopoulos, I., and Kokotović, P., 1995, Nonlinear and Adaptive Control Design (New York: Wiley) 1st edition.

Sussmann, H., Sontag, E., and Yang, Y., 1994, A general result on the stabilization of linear system using bounded controls. IEEE Transactions on Automatic Control, 39, 2411-1425. 